\title{
Prognosis of fracture: evaluation of predictive accuracy of the FRAX algorithm and Garvan nomogram: rejoinder to comments by Pluskiewicz and Drozdzowska
}

\author{
S. K. Sandhu • N. D. Nguyen • J. R. Center • \\ N. A. Pocock • J. A. Eisman • T. V. Nguyen
}

Received: 3 December 2010 / Accepted: 17 December 2010/Published online: 29 January 2011

(C) International Osteoporosis Foundation and National Osteoporosis Foundation 2011

We thank Drs. Pluskiewicz and Drozdzowska for their interest in our work [1] and their thoughtful remarks [2]. We would like to comment on their remarks as follows:

1. We agree that hip fracture is important, and ideally any validation study should consider a separate analysis for hip fracture. However the number of hip fractures was small $(n=20)$ in our study and thus was not sufficient for a full stratified analysis. Despite these small numbers, the concordance for hip fracture prediction between FRAX and Garvan nomogram predicted risk of hip fracture was 0.73 for women and 0.29 for men.

2. The concordance between FRAX and Garvan nomogram predicted probabilities of fracture was generally higher in women than in men. For instance, for any osteoporotic fracture, the correlation between the two algorithms was 0.82 in women but only 0.20 for men.

3. Determining an appropriate risk threshold for treatment depends, among other things, on the effectiveness of treatment and risk-benefit considerations. The latter are, in turn, dependent on the wealth and healthcare system of a country. The National Osteoporosis Foundation recommended thresholds [3] of $20 \%$ for any fracture and 3\% for hip fracture are relevant to the US setting but not necessarily to non-US populations.
We consider that treatment thresholds need further country-specific studies.

The assessment of fracture risk has entered a new era with individualized or absolute risk being the preferred approach. However, the predictive performance of any algorithm should be thoroughly evaluated in relevant cohorts before it is implemented in clinical settings. We thank our colleagues for their thoughtful contribution to the on-going discussion on fracture risk assessment.

\section{References}

1. Sandhu SK, Nguyen ND, Center JR, Pocock NA, Eisman JA, Nguyen TV (2010) Prognosis of fracture: evaluation of predictive accuracy of the FRAX $^{\mathrm{TM}}$ algorithm and Garvan nomogram. Osteoporos Int 21:863-871. doi:10.1007/s00198009-1026-7

2. Pluskiewicz W, Drozdzowska B. Comments on Sandhu et al. Prognosis of fracture: evaluation of predictive accuracy of the FRAX $^{\mathrm{TM}}$ algorithm and Gravan nomogram. Osteoporos Int doi: 10.1007/s00198-010-1526-5

3. National Osteoporosis Foundation (2008) Clinicians guide to prevention and treatment of osteoporosis. Washington DC: National Osteoporosis Foundation

S. K. Sandhu • N. D. Nguyen · J. R. Center N. A. Pocock

J. A. Eisman $\cdot$ T. V. Nguyen $(\square)$

Osteoporosis and Bone Biology Research,

Garvan Institute of Medical Research,

384 Victoria Street,

Sydney, NSW 2010, Australia

e-mail: t.nguyen@garvan.org.au 